\title{
Global metabolomic profiles reveal differences in oxidative stress and inflammation pathways in smokers and moist snuff consumers
}

\author{
Gaddamanugu L. Prasad ${ }^{1 *}$, Bobbette A. Jones ${ }^{1}$, Eckhardt Schmidt ${ }^{1}$, Peter Chen ${ }^{1}$ and Adam D. Kennedy ${ }^{2}$
}

*Correspondence: prasadg@RJRT.com

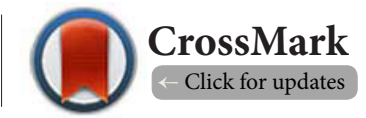

${ }^{1}$ R.J. Reynolds Tobacco Company, P.O. Box 1487, Winston-Salem, NC 27102, USA.

${ }^{2}$ Metabolon, Inc., 617 Davis Dr., Suite 400, Durham, NC 27713, USA.

\begin{abstract}
Background: The existing US epidemiological data show that long-term cigarette smokers are at higher risk of developing serious diseases relative to moist snuff consumers. To understand the effects of tobacco consumption, global metabolomic profiles were generated. Here, we describe metabolomic changes in oxidative stress and inflammation pathways.
\end{abstract}

Methods: Matching plasma, urine, and saliva samples from chronic/long-term smokers (SMK), moist snuff consumers (MSC), and non-tobacco consumers (NTC), 40 subjects in each cohort, were collected in a crosssectional biomarker discovery study. Untargeted metabolomics and data analyses were performed using Metabolon's proprietary technology.

Results: Several biochemicals that significantly differed between study cohorts were identified in all three matrices, with most metabolites found in urine. Random forest analyses of the metabolomes grouped study subjects with a high accuracy and indicated that nicotine and its metabolites primarily drive separation between the NTC and MSC; otherwise, metabolomic profiles of NTC and MSC are more similar to each other, and SMK appear to manifest a distinct metabolomic profile. SMK exhibit lower levels of antioxidants, changes in glutathione metabolism and purine degradation pathways, docosahexaenoate, arachidonate, and 12-hydroxyeicosatetraenoic acid, suggesting increased oxidative stress and inflammation relative to MSC and NTC.

Conclusions: Metabolomic profiles show that while SMK and MSC cohorts exhibit higher levels of nicotine and its metabolites, SMK manifest evidence of increased oxidative stress and inflammation relative to MSC and NTC. These observed biochemical changes in the SMK could be likely due to the combustion-related toxicants present in cigarette smoke.

Impact: Several differentiating metabolites identified herein could be utilized as potential biomarkers of effect. Further, the metabolomic profiles improve our understanding of biological changes in tobacco consumers.

Keywords: Metabolomic profiles, smoking, moist snuff, biomarkers

\section{Introduction}

Chronic smoking exposes smokers to many harmful and potentially harmful constituents and other chemicals in cigarette smoke that are formed during the combustion process $[1,2]$. The local and systemic pathophysiological consequences of smoking have been described at the organ, cellular, and molecular levels [3]. A significant percentage of current smokers, relative to non-smokers, are at higher risk for developing smoking-related diseases such as lung cancer, chronic obstructive pulmonary disease (COPD), cardiovascular disease (CVD), and oral cancer [4]. While smoking cessation continues to be the best approach to reduce harm, the success rates for smoking cessation are 
Prasad et al. Journal of Metabolomics 2015,

rather low [5]. US and European epidemiological studies show that relative to smoking, the health risks associated with the use of smokeless tobacco products (STP) are relatively lower [6]. Therefore, migration from combustible tobacco products to STPs could lower the risk of serious disease for smokers and reduce harm for those who cannot or do not quit smoking $[7,8]$.

A significant body of information exists on the health effects of smoking, but relatively less is known about the effects of the consumption of non-combustible tobacco. Moist snuff is a major category among STPs marketed in USA [9]. Existing epidemiological studies show that the relative risk of several diseases is significantly reduced with moist snuff consumption compared to smoking, although the disease risks are somewhat higher than non-smokers. A number of biomarkers that indicate exposure (termed biomarkers of exposure, BioExp) to nicotine, as well as the toxicants present in smoke and tobacco, have been described $[10,11]$. In contrast, there is limited information on the biomarkers of effect (BioEff) that indicates the effect (s) of exposure to different categories of tobacco products. This gap is particularly notable in understanding the biological effects of STPs and the relevant BioEff.

To gain a better understanding of the chronic health ef fects of moist snuff (used as a representative category for STPs) consumption relative to smoking, we evaluated a fairly large number of previously described potential BioExp and BioEff in two cross-sectional studies. In the first study, the investigation of several CVD biomarkers were evaluated, and differences in some BioExp and BioEff between smok ing and non-smoking cohorts were found [12]. In a second study, which was a biomarker discovery study, the biomarker levels in chronic/long-term smokers (SMK) and moist snuff consumers (MSC) were assessed [53]. Some key findings of that study were: 1) MSC are exposed to higher levels of nicotine and tobacco specific nitrosamines relative to SMK; 2) MSC and non-tobacco consumers (NTC) experienced minimal exposure to combustion-related toxicants; 3) MSC and NTC experienced lower levels of oxidative stress, inflammation, platelet activation, and altered lipid metabolism compared to SMK. Collectively, these findings suggest that MSC and NTC cohorts shared similar biomarker profiles, whereas SMK exhibited distinct biomarker patterns. To further understand the underlying molecular changes due to tobacco consumption, we have also utilized a global approach in our efforts to identify novel BioEff.

Metabolomics is a systematic study of metabolites present in biological samples. The analytical approaches usually employ either nuclear magnetic resonance (NMR) or mass spectrometric methods. Although a relatively newer approach compared to other "omic" technologies, metabolomics is a powerful analytical tool. It has been utilized to evaluate the effect of exposure to smoke constituents [13] and to assess the effect of smoking and smoking cessation [14-18]. In an effort to elucidate biochemical changes in healthy consumers of moist snuff and cigarettes, and to identify potential candidate biomarkers of tobacco effect, we conducted a global metabolomic profiling of plasma, urine, and saliva from the same study cohorts described in the candidate biomarker investigation [53]. The results indicated that the biochemical profile of SMK is distinct from that of MSC and NTC. Between MSC and NTC, the cohorts had fewer metabolic differences in both number and magnitude of biochemicals.

\section{Materials and methods \\ Study conduct}

The clinical conduct of the study was approved by the Independent Investigational Review Board (Plantation, FL). The description of the clinical conduct and sample collections of this cross-sectional study has been described in a separate manuscript [53] and is included in the supplementary section (Supplementary Table S1). Briefly, upon obtaining written informed consent, 40 male subjects (age 35-60 years were enrolled into each consumer group: exclusive long-term smokers (SMK), exclusive moist snuff consumers (MSC), and nontobacco consumers (NTC). Following overnight fasting from tobacco and food, EDTA-plasma and unstimulated saliva (with protease and phosphatase inhibitors) were collected from each subject. Additionally, 24-hour urine samples were collected under ambulatory conditions. Aliquots of the plasma, urine, and saliva samples were used for global metabolomic profiling at Metabolon, Inc. (Durham, NC).

\section{Metabolomic profiling technology}

Metabolomic profiling was performed as described previously for the analysis of saliva, plasma, and urine [19-21]. For all matrices, samples from the three consumer groups were extracted and split into equal parts for analysis on gas chromatography/mass spectrometry and liquid chromatography/mass spectrometry/mass spectrometry platforms [22]. After chromatographic separation, full-scan mass spectrometry (MS) was conducted to record and quantify all detectable ions formed after fragmenting the molecules in each of the samples. Metabolites were identified by matching the ions' chromatographic retention index, nominal mass, and spectral fragmentation signatures to an in-house library of standards for metabolite identification and for metabolite quantitation by peak area integration [23]. For biochemicals that were not covered by the purified standards utilized for the biochemical entries into the library, additional library entries were created based on their unique ion signatures (chromatographic and mass spectral). After this, these unnamed biochemicals could be routinely detected and quantified.

For quality control and run-day performance analysis, labeled internal standards were spiked into all samples at different stages of the data acquisition process. The median relative standard deviations were $6 \%$ and $13 \%$ for plasma, $5 \%$ and $8 \%$ for urine, and $6 \%$ and $10 \%$ for saliva for the internal standards and endogenous biochemicals, respectively. 


\section{Statistical analysis}

Missing values (if any) were assumed to be below the level of detection for that biochemical with the instrumentation used and were imputed with the observed minimum for that particular biochemical. For example, nicotine and its metabolites were below the level of detection in NTC, so they were imputed. After normalization and imputation, the data were log-transformed. Analysis of variance and two-sample $t$-tests (with Welch's correction) were performed to compare data obtained from the plasma, urine, and saliva samples from the three consumer groups. Statistical significance was defined at $p \leq 0.05$. Multiple comparisons were accounted for with the false discovery rate (FDR) method, and each FDR was estimated by q-values. Random forest analysis (RFA) was performed using the R-package "RandomForest" $[24,25]$ and used 50,000 partition trees. The overall error rate for the comparison is listed above the matrix table (Supplementary Section). Binning error, or the out-of-bag (OOB) error rate, is the overall percent error for that particular RFA. For example, if there were 42 subjects out of 120 that were binned incorrectly, the binning error would be $35 \%[(42 / 120) \times 100]$. A lower binning error means the subjects were binned into the correct cohort designation. The greater the binning error, the more the subjects were put into the incorrect cohort designation. In a $3 \times 3$ matrix, random guessing would result in $67 \%$ error. An error rate lower than this would suggest an accurate segregation of the groups by metabolic signature, and the lower the error rate, the more accurate the segregation.

\section{Results}

\section{Global metabolomic profiles of smokers and moist snuff consumers}

Metabolomic profiling of plasma, urine, and saliva from SMK, MSC and NTC resulted in detection of named and unnamed compounds (Table 1; Supplementary Table S2-S4). Comparison by $t$-tests identified many significantly altered biochemicals between the consumer groups. Among the three matrices tested, the highest number of named biochemicals was detected in urine, followed by saliva and plasma. "Unnamed compounds," which did not match any of the chemical identities in Metabolon's biochemical database, were also detected. Relative to NTC and MSC, SMK exhibited the highest

Table 1. Summary of statistical analysis of global metabolomic profiling in tobacco and non-tobacco consumers.

\begin{tabular}{l|lllll}
\hline \multirow{2}{*}{ Matrix } & \multicolumn{3}{|l}{$\begin{array}{l}\text { Metabolites detected } \\
(\boldsymbol{n})\end{array}$} & $\begin{array}{l}\text { Statistically significant analytes } \\
\text { (cohort comparison up/down) }\end{array}$ \\
\cline { 2 - 6 } & Total & $\begin{array}{l}\text { Named/ } \\
\text { Unnamed }\end{array}$ & $\begin{array}{l}\text { SMK vs. } \\
\text { NTC }\end{array}$ & $\begin{array}{l}\text { SMK vs. } \\
\text { MSC }\end{array}$ & $\begin{array}{l}\text { MSC vs. } \\
\text { NTC }\end{array}$ \\
\hline Plasma & 508 & $307 / 201$ & $27 / 41$ & $22 / 57$ & $23 / 15$ \\
Urine & 967 & $423 / 544$ & $78 / 197$ & $80 / 249$ & $49 / 15$ \\
Saliva & 406 & $312 / 94$ & $28 / 66$ & $34 / 57$ & $23 / 23$ \\
\hline
\end{tabular}

SMK: Cigarette smokers; NTC: Non-tobacco consumers;

MSC: Moist snuff consumers number of statistically significant differences $(p<0.05)$ in the levels of named and unnamed biochemicals.

Random forest analyses of the global metabolomic profiles RFA was conducted to determine whether specific metabolites could be identified that predicted the consumer groups. The RFA was performed twice using two datasets (both of which excluded unnamed biochemicals). In the first analysis, nicotine metabolites were identified as strong markers of tobacco exposure when the RFA was performed using all detected metabolites (Supplementary Table S2A). In the second RFA, nicotine metabolites were excluded from the RFA in order to survey the dataset for potential BioEff (Supplementary Table S2B).

For example, analysis of urine metabolomic profiles (Supplementary Table S2A, middle panel) with all metabolites reveals the OOB was at $6.6 \%$ with low class error rates $(\leq 0.1)$, indicating a high accuracy in the separation of study groups. However, with the exclusion of nicotine and its metabolites, the $\mathrm{OOB}$ increased to $42.5 \%$, and the class error rates were $0.1,0.25$, and 0.4 for SMK, NTC, and MSC cohorts, respectively (Supplementary Table S2B, middle panel). This suggests that separation of SMK from the non-smoking cohorts is achieved with or without nicotine and its metabolites, whereas inclusion of nicotine and its metabolites in the RFA determined the accurate binning of MSC. This is illustrated by plotting the prediction percent for urine metabolomic profiles in Figure 1 (using data derived from Supplementary Table S2). Based on the metabolomic profiles, SMK could be predicted with high (90\%) accuracy as SMK with the inclusion or exclusion

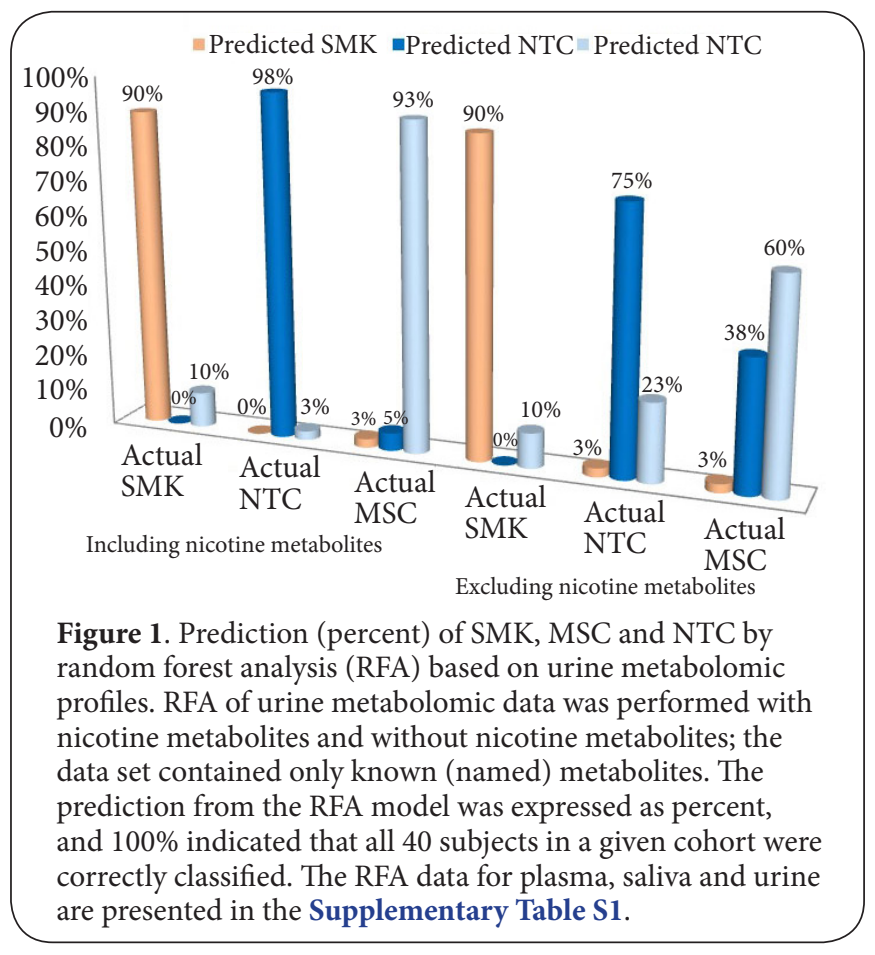


Prasad et al. Journal of Metabolomics 2015,

of nicotine metabolites. The prediction accuracy for NTC and MSC cohorts decreased from $98 \%$ and $93 \%$ with nicotine metabolites to $75 \%$ and $60 \%$, respectively, with the exclusion of nicotine metabolites. Also, more NTC and MSC are incorrectly binned in the absence of nicotine metabolites, as evidenced by higher percentages of predicted MSC (23\%) and NTC (38\%) in the actual NTC and MSC groups, respectively.

Analyses of matching plasma and saliva metabolomes by RFA supported this result (Supplementary Table S2, top and bottom panels, respectively). Collectively, these results suggest that the metabolome of the SMK cohort is distinct from that of the two non-smoking cohorts, and the overall biochemical profiles of NTC and MSC cohorts more closely resemble each other relative to the SMK. Further, nicotine and its metabolites appear to be key determinants of separation between MSC and NTC cohorts, while multiple biochemicals contribute to the distinctiveness of the SMK metabolome.

\section{Biomarkers of exposure: nicotine metabolism}

Nicotine and several of its metabolites were detected in all three matrices of SMK and MSC, reflecting the cohorts' exposure to tobacco (Table 2). Most of these metabolites were detected in urine. Cotinine, the primary metabolite of nicotine, was detected in all the three matrices from SMK and MSC; whereas, nicotine, cotinine- $\mathrm{N}$-oxide, 3-hydroxycotinine, 3-hydroxycotinine $\mathrm{N}$-glucuronide along with two other putative metabolites of nicotine (X-17711 and $\mathrm{X}-17301)$ were found only in urine and saliva. Cotinine, 3-hydroxycotinine, and X-17301 were detected in saliva. Between the tobacco consumer cohorts, MSC exhibited statistically significantly higher levels of several metabolites compared to SMK, while the NTC had baseline levels of cotinine in all three matrices.

\section{Potential biomarkers of effect}

Elevated oxidative stress and chronic inflammation are among the well-described effects of exposure to cigarette smoke. Global metabolomic profiling of plasma, urine, and saliva identified differential levels of several metabolites that are associated with oxidative stress and inflammation pathways, and other physiological pathways in tobacco consumers, particularly in smokers. Here we present data on select metabolomic changes observed in the study.

\section{Oxidative stress}

Several metabolites, implicated in oxidative stress pathways, were observed to be statistically different between the study cohorts (Table 3), with the largest number found in plasma, followed by urine and saliva. The changes reflect the greatest oxidative stress to be present in the SMK group and to a lesser extent in the MSC group. Metabolites that are associated with vitamin metabolism [threonate, $\gamma$-carboxyethyl-hydroxy chroman (CEHC) and a-CEHC glucuronide] and the heme degradation pathway [biliverdin, bilirubin (E,E) and bilirubin $(Z, Z)]$ were statistically significantly decreased in plasma from SMK relative to NTC. SMK, relative to MSC, also exhibited significantly lower levels of threonate across all three matrices, and plasma biliverdin and bilirubin $(Z, Z)$ were also lower in SMK. A similar trend was observed for other metabolites in the MSC cohort, although those changes were not statistically significant. The levels of $\gamma$-tocopherol were, however, higher in SMK and MSC, but reached statistical significance only in SMK. Plasma urate levels in SMK, although not statistically significant, were lower relative to NTC. Urine urate levels were higher in MSC compared to SMK. Interestingly, changes in the levels of these metabolites in MSC and NTC cohorts were not statistically significant in any of the three matrices.

In terms of vitamin metabolism, the levels of threonate, a metabolite of vitamin C (ascorbate), were consistently lower in SMK relative to the other two non-smoking cohorts in all three matrices. Ascorbate was significantly lower in the urine of SMK. Although the statistical cut-off $(p \leq 0.05)$ was missed, urinary ascorbate levels in MSC trended higher than in SMK

Table 2. Biomarkers of exposure: nicotine and its metabolites in tobacco consumers.

\begin{tabular}{lllllllr}
\hline Matrix & Biochemical name & $\begin{array}{l}\text { Fold change } \\
\text { SMK/NTC }\end{array}$ & $\begin{array}{l}\text { P-value } \\
\text { Plasma }\end{array}$ & $\begin{array}{l}\text { Fold change } \\
\text { MSC/NTC }\end{array}$ & $\begin{array}{l}\text { P-value } \\
\text { Urine }\end{array}$ & $\begin{array}{l}\text { Fold change } \\
\text { SMK/MSC }\end{array}$ & $\begin{array}{l}\text { P-value } \\
\end{array}$ \\
& Cotinine & 5.4 & $<0.001$ & 7.1 & $<0.001$ & 0.76 & 0.069 \\
& Cotinine & 6.03 & $<0.001$ & 5.78 & $<0.001$ & 1.04 & 0.343 \\
& 3-Hydroxycotinine & 2.71 & $<0.001$ & 3.69 & $<0.001$ & 0.73 & 0.015 \\
& 3-Hydroxycotinine glucuronide & 4.49 & $<0.001$ & 6.74 & $<0.001$ & 0.52 & $<0.001$ \\
& Cotinine N-oxide & 14.43 & $<0.001$ & 7.19 & $<0.001$ & 0.56 & 0.003 \\
& X-17301 & 3.73 & $<0.001$ & 5.51 & $<0.001$ & 0.68 & 0.053 \\
& X-17711 & 11.1 & $<0.001$ & 14.03 & $<0.001$ & 0.79 & 0.468 \\
& Cotinine & 3.39 & $<0.001$ & 3.9 & $<0.001$ & 0.87 & 0.218 \\
& 3-Hydroxycotinine & 18.31 & $<0.001$ & 22.81 & $<0.001$ & 0.8 & 0.182 \\
& X-17301 & 1.11 & 0.910 & 12.38 & $<0.001$ & 0.09 & $<0.001$ \\
\hline
\end{tabular}

NOTE: Statistically significant differences are highlighted by shading $(\mathrm{P} \leq 0.05)$. The levels of nicotine and its metabolites are below the levels of detection in NTC. The fold changes for nicotine and its metabolites for NTC are calculated based on the imputed values. 
Prasad et al. Journal of Metabolomics 2015,

http://www.hoajonline.com/journals/pdf/2059-0008-1-2.pdf

Table 3. Potential biomarkers of oxidative stress in tobacco consumers.

\begin{tabular}{|c|c|c|c|c|c|c|c|}
\hline Matrix & Biochemical name & $\begin{array}{l}\text { Fold change } \\
\text { SMK/NTC }\end{array}$ & P-value & $\begin{array}{l}\text { Fold change } \\
\text { MSC/NTC }\end{array}$ & P-value & $\begin{array}{l}\text { Fold change } \\
\text { SMK/MSC }\end{array}$ & P-value \\
\hline \multirow[t]{8}{*}{ Plasma } & Threonate & 0.67 & 0.006 & 0.96 & 0.777 & 0.70 & 0.009 \\
\hline & Heme & 1.2 & 0.034 & 1.05 & 0.304 & 1.14 & 0.202 \\
\hline & Bilirubin $(\mathrm{Z}, \mathrm{Z})$ & 0.72 & $<0.001$ & 0.93 & 0.214 & 0.77 & 0.057 \\
\hline & Bilirubin (E,E) & 0.65 & 0.001 & 1.05 & 0.733 & 0.62 & $<0.001$ \\
\hline & Biliverdin & 0.76 & 0.008 & 1 & 0.867 & 0.76 & 0.019 \\
\hline & $\gamma$-Tocopherol & 1.3 & 0.043 & 1.33 & 0.051 & 0.97 & 0.986 \\
\hline & $\gamma$-CEHC & 0.83 & 0.028 & 0.88 & 0.075 & 0.95 & 0.721 \\
\hline & Urate & 0.9 & 0.064 & 1.01 & 0.951 & 0.89 & 0.107 \\
\hline \multirow[t]{4}{*}{ Urine } & Ascorbate & 0.38 & 0.004 & 0.5 & 0.158 & 0.76 & 0.093 \\
\hline & Threonate & 0.74 & 0.001 & 1.03 & 0.876 & 0.72 & 0.001 \\
\hline & a-CEHC glucuronide & 0.55 & 0.004 & 0.81 & 0.191 & 0.68 & 0.134 \\
\hline & Urate & 0.93 & 0.158 & 1.05 & 0.211 & 0.89 & 0.002 \\
\hline \multirow[t]{3}{*}{ Saliva } & Threonate & 0.58 & 0.002 & 0.77 & 0.238 & 0.75 & 0.022 \\
\hline & 5-Oxoproline & 0.71 & 0.274 & 1.01 & 0.5517 & 0.7 & 0.071 \\
\hline & Glutathione (oxidized) & 1.1 & 0.075 & 0.86 & 0.9783 & 1.28 & 0.064 \\
\hline
\end{tabular}

NOTE: Statistically significant differences are highlighted by shading $(\mathrm{P} \leq 0.05)$.

and lower than in NTC.

Another antioxidant vitamin, $\gamma$-tocopherol (a form of vitamin E) was significantly higher in the plasma of SMK, relative to NTC; while MSC also exhibited comparably higher levels of the vitamin, it nearly reached the statistical significance cut-off. Further, the levels of its metabolite, $\gamma$-CEHC in plasma and $\mathrm{a}-\mathrm{CEHC}$ glucuronide (a metabolite of a-tocopherol) in urine, were significantly lower in SMK, relative to NTC, which suggests increased retention/need for the antioxidants in SMK. The levels of these two metabolites were not statistically significant between SMK and MSC, or MSC and NTC. Overall, the MSC and NTC cohorts did not reveal any statistical differences in the levels of the metabolites indicative of oxidative stress. Taken together, the changes in the antioxidants indicate marked changes in the antioxidant capacity in the SMK cohort.

\section{Inflammation}

Several pathways and metabolites which mediate the inflammatory response were found to be significantly altered in SMK relative to the other cohorts. For example, the relative salivary levels of 12-hydroxyeicosatetraenoic acid (12-HETE) (Figure 2A) and plasma levels of docosahexaenoate (DHA) (Figure 2B) were significantly lower in tobacco consumers, and plasma arachidonate was significantly higher in SMK compared to MSC (Figure 2B). Although differences in the levels of these metabolites were statistically significant, the percent changes were modest (below 20\%).

Acceleration in purine degradation and increased oxidative stress is a known indication of chronic inflammation [26,27]. Similarly, several purine degradation metabolites were significantly altered between the study cohorts (Figures 3A and 3B). Plasma levels of hypoxanthine were significantly higher in SMK relative to MSC, and urine xanthine levels were higher in MSC

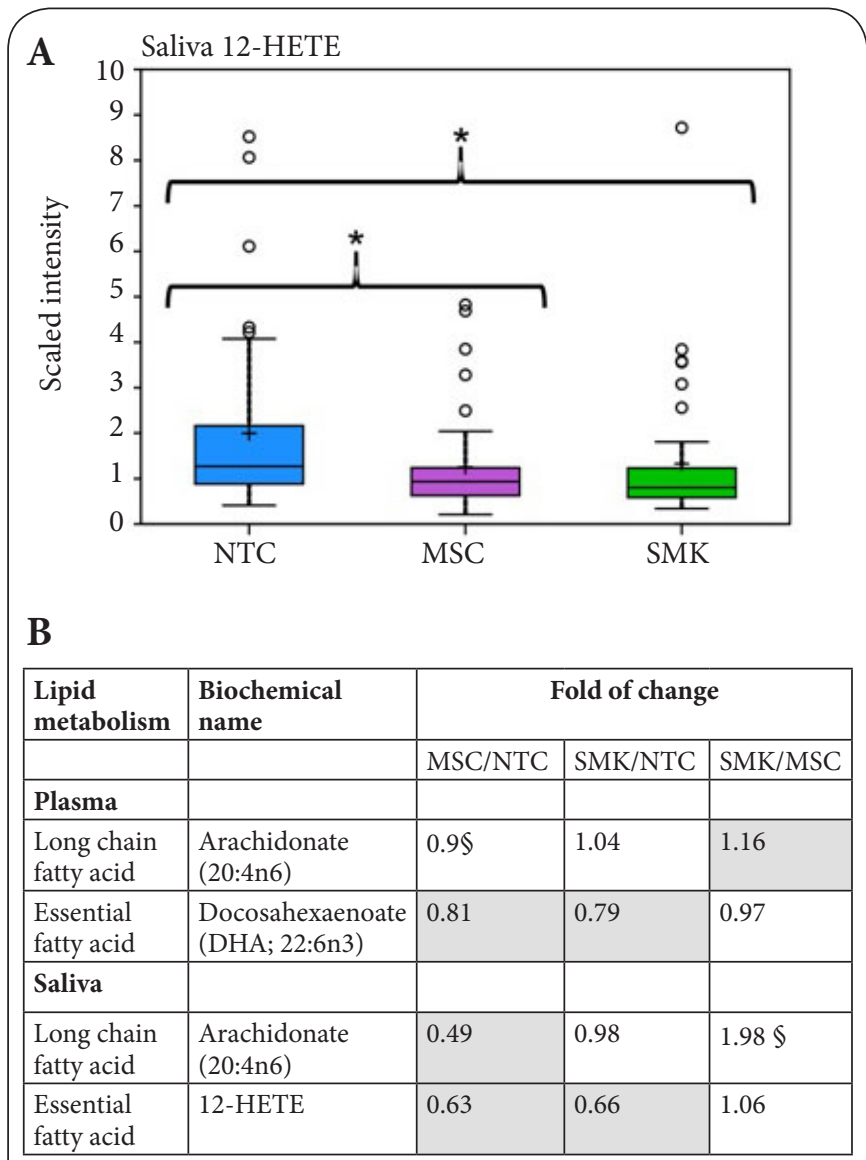

Figure 2. Changes in inflammatory lipid metabolites in tobacco consumers. (A) Salivary 12-HETE significantly decreased in MSC and SMK; (B) Differences in several plasma and salivary lipids were statistically significant. Shaded values indicate $\mathrm{P} \leq 0.05 ; \S$ indicates $0.05<\mathrm{P} \leq 0.1$. 
Prasad et al. Journal of Metabolomics 2015,

A

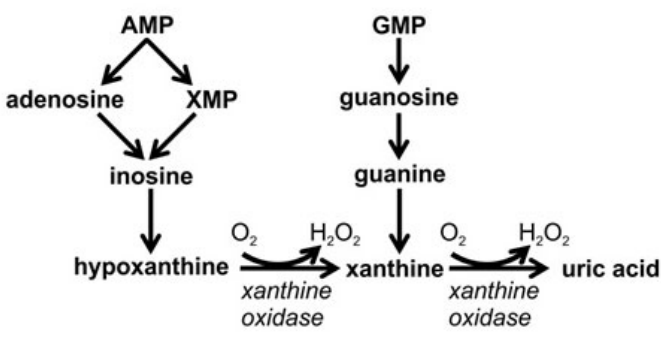

B

\begin{tabular}{|c|c|c|c|c|}
\hline \multirow[t]{2}{*}{ Sub pathway } & \multirow[t]{2}{*}{ Biochemical name } & \multicolumn{3}{|c|}{ Fold of change } \\
\hline & & MSC/NTC & SMK/NTC & SMK/MSC \\
\hline \multicolumn{5}{|l|}{ Plasma } \\
\hline (Hypo)Xanthine/lnosine & Hypoxanthine & 0.88 & 1.08 & 1.22 \\
\hline Guanine & 7-Methylguanine & 1.05 & 1.27 & $1.21 \S$ \\
\hline Urate metabolism & Urate & 1.01 & $0.9 \$$ & 0.89 \\
\hline \multirow[t]{2}{*}{ Uracil } & Uridine & 0.94 & 1.03 & 1.10 \\
\hline & 5-Methyluridine (Ribothymidine) & $0.93 \$$ & 0.97 & 1.04 \\
\hline \multicolumn{5}{|l|}{ Urine } \\
\hline \multirow[t]{4}{*}{ (Hypo)Xanthine } & Xanthine & 0.78 & 0.59 & 0.75 \\
\hline & Xanthosine & 0.94 & 0.83 & $0.88 \$$ \\
\hline & Hypoxanthine & 1.00 & $0.8 \S$ & 0.80 \\
\hline & Inosine & 0.94 & $0.84 \S$ & 0.90 \\
\hline \multirow[t]{4}{*}{ Adenine } & Adenine & 1.01 & $0.88 \S$ & $0.87 \S$ \\
\hline & Adenosine & 1.03 & 0.94 & 0.92 \\
\hline & N1-Methyladenosine & 0.96 & $0.87 \S$ & 0.91 \\
\hline & $\begin{array}{l}\text { Adenosine 3',5'-Cyclic } \\
\text { monophosphate (cAMP) }\end{array}$ & 1.01 & 0.90 & 0.89 \\
\hline \multirow[t]{9}{*}{ Guanine } & Guanine & 0.99 & 1.06 & 1.07 \\
\hline & 7-Methylguanine & 0.99 & 1.06 & 1.07 \\
\hline & Guanidine & 0.99 & 0.76 & 0.77 \\
\hline & N1-Methylguanosine & 1.02 & 0.93 & 0.91 \\
\hline & N2-Methylguanosine & 0.98 & 0.91 & 0.93 \\
\hline & N2, N2-Dimethylguanosine & 1.01 & 0.88 & $0.88 \S$ \\
\hline & N6-Carbamoylthreonyladenosine & 1.00 & 0.88 & $0.88 \$$ \\
\hline & Neopterin & 1.04 & 0.88 & $0.85 \S$ \\
\hline & 7,8-Dihydroneopterin & 0.94 & 0.77 & 0.82 \\
\hline \multirow[t]{2}{*}{ Urate } & Urate & 1.05 & 0.93 & 0.89 \\
\hline & Allantoin & 1.46 & 0.89 & 0.61 \\
\hline \multicolumn{5}{|l|}{ Saliva } \\
\hline \multirow[t]{5}{*}{ (Hypo)Xanthine/lnosine } & Xanthine & 0.93 & 0.84 & 0.91 \\
\hline & Xanthosine & 0.75 & $0.68 \$$ & 0.9 \\
\hline & Hypoxanthine & $0.68 \S$ & 0.71 & 1.04 \\
\hline & Inosine & 0.76 & 1.14 & 1.51 \\
\hline & 2'-Deoxyinosine & 0.74 & 0.97 & 1.32 \\
\hline \multirow[t]{6}{*}{ Adenine } & Adenine & 0.96 & 1.14 & 1.19 \\
\hline & Adenosine & 0.93 & 1.73 & 1.86 \\
\hline & 1-Methyladenine & 0.86 & 0.63 & 0.72 \\
\hline & N1-Methyladenosine & 0.66 & 1.07 & 1.63 \\
\hline & Adenosine 2'-Monohosphate (2'-AMP) & 0.7 & 0.9 & 1.29 \\
\hline & Adenosine 5'-Monophosphate (AMP) & 0.56 & 0.44 & 0.79 \\
\hline \multirow[t]{4}{*}{ Guanine } & Guanine & 0.6 & 0.57 & 0.95 \\
\hline & Guanosine & $0.79 \$$ & $1.23 \S$ & 1.57 \\
\hline & 2'-Deoxyguanosine & 0.81 & 0.99 & 1.22 \\
\hline & N6-Carbamoylthreonyladenosine & 1.01 & 1.09 & 1.08 \\
\hline \multirow[t]{2}{*}{ Urate } & Urate & 1.03 & 0.92 & 0.9 \\
\hline & Allantoin & 0.95 & 0.86 & 0.91 \\
\hline
\end{tabular}

Figure 3. Changes in purine metabolites associated with oxidative stress and inflammation in tobacco consumers.

(A) Pathway diagram of purine degradation and generation of oxidative stress.

(B) Statistical results showing differences in purine metabolism between cohort groups in plasma, urine, and saliva. Shaded values indicate $\mathrm{P} \leq 0.05 ; \S$ indicates $0.05<\mathrm{P} \leq 0.1$. 
and NTC relative to SMK. Further, saliva levels of adenosine were elevated in SMK, while guanine levels were lower. Collectively, these findings suggest that chronic smoking-induced inflammatory responses are detectable in healthy smokers, while such responses are less pronounced, if detectable, in MSC relative to NTC.

\section{Discussion}

We performed this study with a goal to understand the underlying biochemical changes in long-term tobacco consumers and to discover novel biomarkers of tobacco effect. The results of untargeted metabolomic profiling of matching plasma, urine, and saliva, carried out for the first time, reveal detailed metabolomic changes in a cohort of tobacco consumers and non-consumers. The key findings of this study were: a) overall, SMK exhibited distinct biochemical profiles relative to MSC and NTC, with the latter two groups showing more similar profiles; b) SMK exhibited more perturbations in oxidative stress, inflammation, and other pathways relative to MSC. Vitamin and purine metabolism were some of the prominent changes associated with chronic smoking that were observed. In addition, many biochemical changes in plasma, urine, and saliva, which mapped into well-defined physiological pathways, were concordantly and markedly altered due to smoking, and to a lesser degree with consumption of moist snuff.

Untargeted metabolomic profiling is a powerful tool employed in biomarker research [28] and to gain a better understanding of the effects of nutritional and environmental exposure [29,30], critical path [31], and for other purposes. Several researchers have utilized metabolomic profiling to evaluate the effects of smoking through NMR-based [14] and MS-based technologies $[15,32]$. In pilot experiments, the MSbased metabolomics yielded higher numbers of metabolites (this study) than NMR-based methods (unpublished data).

Several researchers have shown that smokers and nonsmokers could be readily distinguished based on their metabolomic profiles [14,15], and those analyses included nicotine and its metabolites. We were able to replicate those results in our study as well. Here, we report a greater coverage of the metabolome in tobacco consumers and present data on BioExp and BioEff. Given the large number of metabolites detected in the three metabolomes and the diverse physiological pathways into which they map, this manuscript is focused on BioExp and BioEff in pathways of oxidative stress and inflammation.

Nicotine metabolites, including cotinine $\mathrm{N}$-oxide, 3-hydroxy cotinine, and cotinine, were the top metabolites contributing to the separation of the groups. Further, RFA of the global profiles clearly distinguished SMK from NTC and MSC, independent of the inclusion of known nicotine metabolites in the analyses. Exclusion of nicotine metabolites from the analyses, however, increased the error in distinguishing MSC and NTC cohorts, suggesting the overall resemblance of metabolic profiles of MSC and NTC. Inclusion of unknown metabolites also yielded similar results (data not shown).
Matching plasma, urine, and saliva samples were collected in a biomarker discovery study [53] and many established BioExp, including nicotine and its metabolites, were evaluated. The metabolomic profiling detected several nicotine metabolites, with the largest number found in SMK and MSC urine. The relative levels of the BioExp and BioEff (described in the literature) detected in the targeted assays and metabolomic profiling are directionally concordant. For example, both methods showed that the levels of cotinine, a major metabolite of nicotine, were higher in MSC, compared to SMK. Further, $X-17301$, a compound with a likely structure of 6-hydroxycotinine, based on retention time and mass spectra (Supplementary Figure $\mathbf{S 1}$ ), is highly enriched in saliva of MSC. Overall, the results from both methods indicate a potentially higher exposure to nicotine in the MSC group, a finding consistent with published data [33].

Our data also showed that several metabolites reflecting antioxidant capacity were significantly altered in SMK, but not MSC, when compared to NTC. In one antioxidant system, an increase in heme degradation by hemeoxygenase (HO-1) to biliverdin and bilirubin is a protective anti-inflammatory response to increased oxidative stress. Exposure to cigarette smoke (or its constituents) is known to increase the expression of HO-1 [34-37]. Plasma metabolomic profiles showed increased levels of heme, and decreased levels of bilirubin and biliverdin in SMK, relative to NTC, indicating a possible impairment of the heme degradation pathway. Consistent with our findings, serum bilirubin levels have been reported be lower in active smokers [38].

Other changes in vitamin metabolism included consistently decreased levels of threonate in plasma, urine, and saliva of SMK compared to NTC, and lower urine levels of vitamin C, which are in agreement with published literature [39]. Taken together with a decrease in urine a-CEHC glucourinide and urate (although not statistically significant), these changes underscore an altered antioxidant metabolism in smokers [40]. Urine ascorbate levels were relatively lower in MSC compared to SMK, but did not reach statistical significance. Further, it has been reported that plasma and gingival crevicular fluids contain lower levels of vitamin $C$ and vitamin $E$ in smokers [41]. These data are in agreement with published findings that smokers exhibit lower levels of plasma antioxidants (but elevated $\gamma$-tocopherol) independent of dietary intake of antioxidants [42]. Some in vitro studies indicated that exposure to moist snuff extracts causes oxidative stress [43], and antioxidants protect cells from the effects of moist snuff [44].

Glutathione oxidation has been reported to be elevated in cigarette smokers [45]. Our metabolomic profiling found that oxidized glutathione in saliva was slightly elevated but the difference did not meet the statistical cutoff ( $p \leq 0.05$ ) (Table 3 ), while 5-oxoproline levels were lower in SMK relative to MSC. In MSC, however, these metabolites were mostly similar to those found in NTC. The plasma and urine levels of these metabolites in the metabolomic analyses, or measurement 
Prasad et al. Journal of Metabolomics 2015,

of oxidized and reduced glutathione in targeted assays [53] did not reveal any differences among the study cohorts.

Collectively, data on several antioxidant levels in the study groups and the metabolite data presented herein indicated that subjects who chronically smoked (SMK) had increased oxidative stress. MSC demonstrated detectable changes in the oxidative stress-related metabolites, but the changes were muted and not significantly different from the levels found in NTC.

Several lipid changes in plasma and saliva could be related to mediators of the inflammatory response. The decreased plasma DHA in SMK and MSC suggest impaired antioxidant and anti-inflammatory ability [46]. Arachidonic acid metabolism is a key pathway in regulation of inflammatory responses, and several studies, including our data [53] have shown elevated levels of isoprostanes, lipoxygenase metabolites such as 12 HETE, and leukotrienes in smokers [10,47-49]. Metabolomic profiles presented herein show changes in the levels of arachidonic acid in plasma and saliva and salivary 12-HETE (Figure 2) among the study groups. Decreased levels of 12 HETE in saliva of tobacco consumers (SMK and MSC) relative to NTC was noted in this study. Although a previous study did not find differences in the levels of 12-HETE between smokers and non-smokers, several other eicosanoids were found to be lower in smokers [50]. While elevated levels of serum 12-HETE were found to be associated with several types of cancer, increased salivary levels of 12-HETE were detected in oral cancer patients. Together with elevated arachidonic acid, higher levels of 12-HETE were suggested to be markers of inflammation associated with oral cancer [51]. Although, elevated plasma levels of arachidonic acid were found in SMK, relative to MSC, salivary arachidonate levels were lower in MSC $(p \leq 0.05)$ relative to NTC, and trended in that direction compared to SMK ( $p 0.05 \leq p<0.1$ ).

Oxidative stress is implicated in purine degradation, which in turn plays a role in the inflammatory process (Figure $3 \mathrm{~A}$ ). Several studies have shown adenosine and other purines to be elevated in COPD. Further, purine degradation products such as adenosine, hypoxanthine, and inosine were present at higher levels in gingival crevicular fluid from patients with periodontal disease $[19,26]$ and in saliva of smokers [15]. Data presented herein showed elevated levels of hypoxanthine (plasma) and a corresponding lower urinary hypoxanthine, as well as elevated levels of adenosine and inosine in saliva of SMK relative to the MSC (Figure 3B). Further, the levels of urinary urate, which is known to possess free-radical scavenging properties, was lower in SMK (vs NTC) and achieved a statistical significance when compared to MSC. Thus, subjects in the SMK group exhibited altered hypoxanthine oxidation and a lower excretion of urate, both of which could potentiate inflammation.

The global metabolomic profiling also detected a diverse array of metabolites, which included tobacco/smoke constituents, metabolites of nicotine, those altered in response to tobacco exposure (potential biomarkers of tobacco effect), microbial metabolites, and those derived from dietary sources (to be communicated in a separate publication). The potential sources of some of the likely tobacco-related metabolites were discussed [15]. While the dietary status of the study participants could influence the levels of some of the biomarkers, particularly those related to nutritional status (e.g., vitamin metabolism), all the subjects appeared to be well nourished in terms of their caloric intake and other nutrients. Significant differences were noted in SMK intake of alcohol, caffeine, fatty acid, and energy derived from fat, as determined by the National Cancer Institute's Diet History Questionnaire (http://riskfactor.cancer.gov/DHQ/) (Supplementary Table S6).

There are several strengths to the global profiling data that are presented herein. Some of the candidate biomarkers identified were also evaluated using targeted assays [53]. Comparison of the results between global metabolomic profiling and the targeted assays from the same tobacco consumers revealed very similar data. For example, exposure to nicotine and nicotine metabolites was increased in MSC relative to SMK, however, elevated oxidative stress and inflammation were seen in SMK. Further, data from plasma, urine, and saliva revealed concordant changes in metabolites/ pathways implicated in oxidative stress and inflammation. Our data are consistent with the established and generally accepted findings that smokers experience elevated oxidative stress and a chronic inflammatory state. The MSC generally appear to resemble the NTC in their metabolomic profiles, although more moderate perturbations in the oxidative stress and inflammation pathways are seen in these data. Similar changes in other physiological pathways in tobacco consumers were noted, and those data will be presented in a separate follow-up communication.

Independent qualification and validation is a critical element in translating the differentiating metabolites detected in metabolomic profiling into potential biomarkers [52]. Many of the BioExp (nicotine and its metabolites and the combustion biomarkers) have been validated in smokers and non-tobacco consumers $[10,11]$, and in moist snuff consumers as well [53]. Qualification of other differentiating metabolites reported herein is in progress.

Longitudinal and case-control studies will provide additional information on the mechanistic aspects of time dependent changes leading to diseases associated with tobacco consumption. The scope of this cross-sectional study is to achieve a fundamental understanding of the molecular and physiological perturbations in healthy long-term tobacco consumers. Such perturbations, in susceptible individuals, could potentially lead to a disease state. Thus, the metabolomic changes reported herein provide useful insights into underlying physiological changes relating to oxidative stress and inflammation in smokers and moist snuff consumers and may serve as potential BioEff.

\section{Conclusions}

Untargeted global metabolomic profiles of biofluids, for the 
Prasad et al. Journal of Metabolomics 2015,

first time, revealed marked differences between healthy tobacco consumers, suggesting the differences in the physiological impact of different tobacco products. While the BioExp (nicotine and some of its metabolites) demonstrated exposure to tobacco, significant and consistent differences were observed in metabolites associated with oxidative stress, inflammation, and lipid metabolism in plasma, urine, and saliva of SMK, MSC and NTC, with the latter two groups generally being more similar to each other than to the SMK cohort. Several of the changes in the significant metabolites were reproducible in targeted assays and uncovered new potential biomarker candidates. The metabolite changes in SMK reported herein were consistent with those reported by other investigators who utilized different metabolomic platforms or samples from different cohorts $[\mathbf{1 4}, \mathbf{1 5 , 5 4 ]}$. Thus, metabolomics is a useful technique to elucidate the pathophysiological changes in smokers and consumers of other tobacco products.

List of abbreviations

12-HETE: 12-hydroxyeicosatetraenoic acid

BioEff: Biomarkers of effect

BioExp: Biomarkers of exposure

COPD: Chronic obstructive pulmonary disease

CECH: Carboxyethyl-hydroxychroman

CVD: Cardiovascular disease

DHA: Docosahexaenoate

FDR: False discovery rate

HO-1: Heme oxygenase-1

MS: Mass spectrometry

MSC: Moist snuff consumers

NMR: Nuclear magnetic resonance

NTC: Non-tobacco consumers

OOB: Out of bag

RFA: Random forest analysis

SMK: Cigarette smokers

STP: Smokeless tobacco products

\section{Additional files}

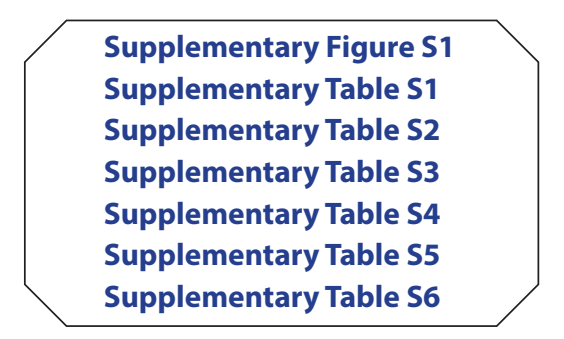

Competing interests

The authors declare that they have no competing interests.

\section{Authors' contributions}

\begin{tabular}{|l|c|c|c|c|c|}
\hline Authors' contributions & GLP & BAJ & ES & PC & ADK \\
\hline Research concept and design & $\checkmark$ & $\checkmark$ & -- & -- & -- \\
\hline Collection and/or assembly of data & $\checkmark$ & -- & $\checkmark$ & -- & $\checkmark$ \\
\hline Data analysis and interpretation & $\checkmark$ & -- & -- & -- & $\checkmark$ \\
\hline Writing the article & $\checkmark$ & -- & -- & $\checkmark$ & $\checkmark$ \\
\hline Critical revision of the article & $\checkmark$ & $\checkmark$ & -- & -- & $\checkmark$ \\
\hline Final approval of article & $\checkmark$ & $\checkmark$ & $\checkmark$ & $\checkmark$ & $\checkmark$ \\
\hline Statistical analysis & -- & -- & -- & -- & $\checkmark$ \\
\hline
\end{tabular}

\section{Acknowledgement}

This study was funded by R.J. Reynolds Tobacco Company (RJRT). ClinicalTrials.gov Identifier: NCT01923402.

\section{Publication history}

Editor: Philip D. Cotter, Pacific Diagnostics Clinical Laboratory, USA. Received: 07-Jul-2015 Final Revised: 11-Aug-2015

Accepted: 31-Aug-2015 Published: 09-Sep-2015

\section{References}

1. Rodgman A and Perfetti TA. The Chemical Components of Tobacco and Tobacco Smoke. In. Second Edition. Aufl. Boca Raton, FL: CRC Press. 2013; 1784. | Book

2. Food and Drug Administration HHS. Harmful and Potentially Harmful Constituents in Tobacco Products and Tobacco Smoke; Established List. Federal Register. 2012; 20034-20037. I Pdf

3. Centers for Disease Control and Prevention. How Tobacco Smoke Causes Disease: The Biology and Behavioral Basis for Smoking-Attributable Disease: A Report of the Surgeon General.Publications and Reports of the Surgeon General. Atlanta (GA). 1-727. I Pdf

4. Mattson ME, Pollack ES and Cullen JW. What are the odds that smoking will kill you? Am J Public Health. 1987; 77:425-31. I PubMed Abstract | PubMed Full Text

5. American Lung Association. Trends in Tobacco Use. 2011; 1-44. | Pdf

6. Lee PN and Hamling J. The relation between smokeless tobacco and cancer in Northern Europe and North America. A commentary on differences between the conclusions reached by two recent reviews. BMC Cancer. 2009; 9:256. | Article | PubMed Abstract | PubMed Full Text

7. Hatsukami DK, Slade J, Benowitz NL, Giovino GA, Gritz ER, Leischow S and Warner KE. Reducing tobacco harm: research challenges and issues. Nicotine Tob Res. 2002; 4 Suppl 2:S89-101. | Article I PubMed

8. Zeller M and Hatsukami D. The Strategic Dialogue on Tobacco Harm Reduction: a vision and blueprint for action in the US. Tob Control. 2009, 18:324-32. | Article | PubMed

9. Borgerding MF, Bodnar JA, Curtin GM and Swauger JE. The chemical composition of smokeless tobacco: a survey of products sold in the United States in 2006 and 2007. Regul Toxicol Pharmacol. 2012; 64:36787. | Article | PubMed

10. Calapai G, Caputi AP, Mannucci C, Gregg EO, Pieratti A, Aurora Russo G, Chaudhary N, Puntoni R, Lowe F, McEwan M, Bassi A, Morandi S and Nunziata A. A cross-sectional investigation of biomarkers of risk after a decade of smoking. Inhal Toxicol. 2009; 21:1138-43. | Article I PubMed

11. Roethig HJ, Munjal S, Feng S, Liang Q, Sarkar M, Walk RA and Mendes PE. Population estimates for biomarkers of exposure to cigarette smoke in adult U.S. cigarette smokers. Nicotine Tob Res. 2009; 11:1216-25. | Article | PubMed

12. Nordskog BK, Brown BG, Marano KM, Campell LR, Jones BA and Borgerding MF. Study of cardiovascular disease biomarkers among tobacco consumers, part 2: biomarkers of biological effect. Inhal Toxicol. 2015; 27:157-66. | Article | PubMed Abstract | PubMed Full Text

13. Vulimiri SV, Misra M, Hamm JT, Mitchell M and Berger A. Effects of mainstream cigarette smoke on the global metabolome of human lung epithelial cells. Chem Res Toxicol. 2009; 22:492-503. | Article | PubMed

14. Hsu PC, Zhou B, Zhao Y, Ressom HW, Cheema AK, Pickworth W and Shields PG. Feasibility of identifying the tobacco-related global metabolome in blood by UPLC-QTOF-MS. J Proteome Res. 2013; 12:679-91. | Article | PubMed Abstract | PubMed Full Text

15. Mueller DC, Piller M, Niessner R, Scherer M and Scherer G. Untargeted metabolomic profiling in saliva of smokers and nonsmokers by a validated GC-TOF-MS method. J Proteome Res. 2014; 13:1602-13. | Article | PubMed

16. Vulimiri SV, Misra M, Hamm JT and Berger A. Effect of mainstream cigarette smoke gas/vapor phase and wet total particulate matter on the metabolic pathways of human lung epithelial cells: The 'metabolomic' approach. 47th Annual Meeting of the Society of Toxicology Seattle, WA; 2008. I Pdf 
Prasad et al. Journal of Metabolomics 2015,

17. Wang-Sattler R, Yu Y, Mittelstrass K, Lattka E, Altmaier E, Gieger C, Ladwig KH, Dahmen N, Weinberger KM, Hao P, Liu L, Li Y, Wichmann HE, Adamski J, Suhre $\mathrm{K}$ and Illig T. Metabolic profiling reveals distinct variations linked to nicotine consumption in humans--first results from the KORA study. PLoS One. 2008; 3:e3863. | Article | PubMed Abstract | PubMed Full Text

18. Xu T, Holzapfel C, Dong X, Bader E, Yu Z, Prehn C, Perstorfer K, Jaremek M, Roemisch-MargI W, Rathmann W, Li Y, Wichmann HE, Wallaschofski H, Ladwig KH, Theis F, Suhre K, Adamski J, Illig T, Peters A and Wang-Sattler R. Effects of smoking and smoking cessation on human serum metabolite profile: results from the KORA cohort study. BMC Med. 2013; 11:60. | Article | PubMed Abstract | PubMed Full Text

19. Barnes VM, Kennedy AD, Panagakos F, Devizio W, Trivedi HM, Jonsson T, Guo L, Cervi S and Scannapieco FA. Global metabolomic analysis of human saliva and plasma from healthy and diabetic subjects, with and without periodontal disease. PLoS One. 2014; 9:e105181. | Article | PubMed Abstract | PubMed Full Text

20. Gall WE, Beebe K, Lawton KA, Adam KP, Mitchell MW, Nakhle PJ, Ryals JA, Milburn MV, Nannipieri M, Camastra S, Natali A and Ferrannini E. alphahydroxybutyrate is an early biomarker of insulin resistance and glucose intolerance in a nondiabetic population. PLoS One. 2010; 5:e10883. Article | PubMed Abstract | PubMed Full Text

21. Ganti S, Taylor SL, Kim K, Hoppel CL, Guo L, Yang J, Evans C and Weiss RH. Urinary acylcarnitines are altered in human kidney cancer. Int $\mathrm{J}$ Cancer. 2012; 130:2791-800. | Article | PubMed Abstract | PubMed Full Text

22. Evans AM, DeHaven CD, Barrett T, Mitchell M and Milgram E. Integrated, nontargeted ultrahigh performance liquid chromatography/electrospray ionization tandem mass spectrometry platform for the identification and relative quantification of the small-molecule complement of biological systems. Anal Chem. 2009; 81:6656-67. | Article | PubMed

23. Dehaven CD, Evans AM, Dai $H$ and Lawton KA. Organization of GC/MS and LC/MS metabolomics data into chemical libraries. J Cheminform. 2010; 2:9. | Article | PubMed Abstract | PubMed Full Text

24. Breiman L. Random Forest. Mach Learn. 2001: 45:5-32. | Article

25. Liaw $A$ and Wiener M. Classification and regression by random forest. $R$ News. 2002: 2:18-22. I Pdf

26. Barnes VM, Teles R, Trivedi HM, Devizio W, Xu T, Mitchell MW, Milburn $\mathrm{MV}$ and Guo $\mathrm{L}$. Acceleration of purine degradation by periodontal diseases. J Dent Res. 2009; 88:851-5. | Article | PubMed

27. Miesel $R$ and Zuber $M$. Elevated levels of xanthine oxidase in serum of patients with inflammatory and autoimmune rheumatic diseases. Inflammation. 1993; 17:551-61. | Article | PubMed

28. Cox DG, Oh J, Keasling A, Colson KL and Hamann MT. The utility of metabolomics in natural product and biomarker characterization. Biochim Biophys Acta. 2014; 1840:3460-3474. | Article | PubMed

29. Jones DP, Park $Y$ and Ziegler TR. Nutritional metabolomics: progress in addressing complexity in diet and health. Annu Rev Nutr. 2012; 32:183202. | Article | PubMed Abstract | PubMed Full Text

30. Miller GW and Jones DP. The nature of nurture: refining the definition of the exposome. Toxicol Sci. 2014; 137:1-2. | Article | PubMed Abstract | PubMed Full Text

31. Schnackenberg LK and Beger RD. Metabolomic biomarkers: their role in the critical path. Drug Discov Today Technol. 2007; 4:13-6. | Article | PubMed

32. Cross AJ, Boca S, Freedman ND, Caporaso NE, Huang WY, Sinha R, Sampson JN and Moore SC. Metabolites of tobacco smoking and colorectal cancer risk. Carcinogenesis. 2014; 35:1516-22. | Article | PubMed Abstract | PubMed Full Text

33. Hecht SS, Carmella SG, Murphy SE, Riley WT, Le C, Luo X, Mooney M and Hatsukami DK. Similar exposure to a tobacco-specific carcinogen in smokeless tobacco users and cigarette smokers. Cancer Epidemiol Biomarkers Prev. 2007; 16:1567-72. | Article | PubMed

34. Almolki A, Guenegou A, Golda S, Boyer L, Benallaoua M, Amara N, Bachoual R, Martin C, Rannou F, Lanone S, Dulak J, Burgel PR, El-Benna J, Leynaert B, Aubier M and Boczkowski J. Heme oxygenase-1 prevents airway mucus hypersecretion induced by cigarette smoke in rodents and humans. Am J Pathol. 2008; 173:981-92. | Article | PubMed Abstract I PubMed Full Text
35. Baglole CJ, Sime PJ and Phipps RP. Cigarette smoke-induced expression of heme oxygenase- 1 in human lung fibroblasts is regulated by intracellular glutathione. Am J Physiol Lung Cell Mol Physiol. 2008; 295:L624-36. | Article | PubMed Abstract | PubMed Full Text

36. Goven D, Boutten A, Lecon-Malas V, Marchal-Somme J, Soler P, Boczkowski J and Bonay M. Induction of heme oxygenase-1, biliverdin reductase and $\mathrm{H}$-ferritin in lung macrophage in smokers with primary spontaneous pneumothorax: role of HIF-1alpha. PLoS One. 2010; 5:e10886. | Article | PubMed Abstract | PubMed Full Text

37. Kim HP, Wang X, Chen ZH, Lee SJ, Huang MH, Wang Y, Ryter SW and Choi AM. Autophagic proteins regulate cigarette smoke-induced apoptosis: protective role of heme oxygenase-1. Autophagy. 2008; 4:887-95. | Article I PubMed

38. Zucker SD, Horn PS and Sherman KE. Serum bilirubin levels in the U.S. population: gender effect and inverse correlation with colorectal cancer. Hepatology. 2004; 40:827-35. | Article | PubMed

39. Schectman G, Byrd JC and Gruchow HW. The influence of smoking on vitamin C status in adults. Am J Public Health. 1989; 79:158-62. | PubMed Abstract | PubMed Full Text

40. Bruno RS and Traber MG. Cigarette smoke alters human vitamin $\mathrm{E}$ requirements. J Nutr. 2005; 135:671-4. | Article | PubMed

41. Seri M, D'Alessandro A and Seri S. The effect of cigarette smoking on vitamin C and vitamin E levels of gingival crevicular fluid. Boll Soc Ital Biol Sper. 1999; 75:21-5. | PubMed

42. Dietrich M, Block G, Norkus EP, Hudes M, Traber MG, Cross CE and Packer L. Smoking and exposure to environmental tobacco smoke decrease some plasma antioxidants and increase gamma-tocopherol in vivo after adjustment for dietary antioxidant intakes. Am J Clin Nutr. 2003; 77:1606. | Article | PubMed

43. Mitchell C, Joyce AR, Piper JT, McKallip RJ and Fariss MW. Role of oxidative stress and MAPK signaling in reference moist smokeless tobaccoinduced HOK-16B cell death. Toxicol Lett. 2010; 195:23-30. | Article | PubMed

44. Bagchi M, Balmoori J, Bagchi D, Ray SD, Kuszynski C and Stohs SJ. Smokeless tobacco, oxidative stress, apoptosis, and antioxidants in human oral keratinocytes. Free Radic Biol Med. 1999; 26:992-1000. | Article | PubMed

45. Giuca MR, Giuggioli E, Metelli MR, Pasini M, lezzi G, S DE and Tripodi D. Effects of cigarette smoke on salivary superoxide dismutase and glutathione peroxidase activity. J Biol Regul Homeost Agents. 2010; 24:359-66. | PubMed

46. Cipollina C, Di Vincenzo S, Gerbino S, Siena L, Gjomarkaj M and Pace E. Dual anti-oxidant and anti-inflammatory actions of the electrophilic cyclooxygenase-2-derived 17-oxo-DHA in lipopolysaccharide- and cigarette smoke-induced inflammation. Biochim Biophys Acta. 2014; 1840:2299-309. | Article | PubMed

47. Duffield-Lillico AJ, Boyle JO, Zhou XK, Ghosh A, Butala GS, Subbaramaiah K, Newman RA, Morrow JD, Milne GL and Dannenberg AJ. Levels of prostaglandin E metabolite and leukotriene $E(4)$ are increased in the urine of smokers: evidence that celecoxib shunts arachidonic acid into the 5-lipoxygenase pathway. Cancer Prev Res (Phila). 2009; 2:322-9. | Article | PubMed Abstract | PubMed Full Text

48. Liu J, Liang Q, Frost-Pineda K, Muhammad-Kah R, Rimmer L, Roethig H, Mendes $P$ and Sarkar M. Relationship between biomarkers of cigarette smoke exposure and biomarkers of inflammation, oxidative stress, and platelet activation in adult cigarette smokers. Cancer Epidemiol Biomarkers Prev. 2011; 20:1760-9. | Article | PubMed

49. Seet RC, Lee CY, Loke WM, Huang SH, Huang H, Looi WF, Chew ES, Quek AM, Lim EC and Halliwell B. Biomarkers of oxidative damage in cigarette smokers: which biomarkers might reflect acute versus chronic oxidative stress? Free Radic Biol Med. 2011; 50:1787-93. | Article | PubMed

50. Wu-Wang CY, Wang SL, Lim C, Milles M, Slomiany A and Slomiany BL. Cigarette smoking reduces human salivary eicosanoids. Prostaglandins Leukot Essent Fatty Acids. 1992; 47:101-4. | Article | PubMed

51. Metzger K, Angres G, Maier H and Lehmann WD. Lipoxygenase products in human saliva: patients with oral cancer compared to controls. Free Radic Biol Med. 1995; 18:185-94. | Article | PubMed 
Prasad et al. Journal of Metabolomics 2015,

http://www.hoajonline.com/journals/pdf/2059-0008-1-2.pdf

52. Koulman A, Lane GA, Harrison SJ and Volmer DA. From differentiating metabolites to biomarkers. Anal Bioanal Chem. 2009; 394:663-70. | Article | PubMed Abstract | PubMed Full Text

53. Prasad GL, Jones BA, Chen P and Gregg EO. A cross-sectional study of biomarkers of exposure and effect in smokers and moist snuff consumers. Clin Chem Lab Med. In Press. 2015.

54. Cross AJ, Boca S, Freedman ND, Caporaso NE, Huang WY, Sinha R, Sampson JN and Moore SC. Metabolites of tobacco smoking and colorectal cancer risk. Carcinogenesis. 2014; 35:1516-22. | Article | PubMed Abstract | PubMed Full Text

\section{Citation:}

Prasad GL, Jones BA, Schmidt E, Chen P and Kennedy AD. Global metabolomic profiles reveal differences in oxidative stress and inflammation pathways in smokers and moist snuff consumers. J Metabolomics. 2015; 1:2. http://dx.doi.org/10.7243/2059-0008-1-2 